\title{
A change in human faecal flora in response to inclusion of gum arabic in the diet
}

\author{
BY G. M. WYATT, C. E. BAYLISS AND J. D. HOLCROFT* \\ Agricultural and Food Research Council, Food Research Institute Norwich, Colney Lane, \\ Norwich, NR4 $7 U A$
}

(Received 9 April 1985 - Accepted 16 October 1985)

\begin{abstract}
1. Gum arabic is a water-soluble polysaccharide resistant to human gut enzymes and thus can be described as dietary fibre.

2. Using a most-probable-number technique, estimates were made of total anaerobes and of gum-arabic fermenters in the faeces of a volunteer during a control period and during addition of $10 \mathrm{~g}$ gum arabic/d to the diet. Using an enrichment technique, the principal bacteria able to utilize gum arabic as the only carbohydrate source were isolated and characterized.

3. Faecal samples were analysed for undegraded gum arabic and, following acid-hydrolysis, for total sugars.

4. The proportion of the faecal flora able to degrade the gum arabic polymer rose from an initial level of $6.5 \%$ to more than $50 \%$ during gum-arabic ingestion, and subsequently returned to the control level after ingestion ceased. The principal gum-arabic fermenters were species of Bacteroides and Bifidobacterium.

5. Undegraded gum arabic was not detected in any faecal sample nor were there significant differences in the level of total sugars in acid-hydrolysed faeces between gum arabic and control periods.

6. The results presented indicate a direct and rapid change in faecal flora in response to a specific change in the diet of a human volunteer.
\end{abstract}

Little change in human faecal flora has been detected following changes in diet (Moore et al. 1981; Savage, 1982) and studies have been complicated by the differences in flora seen between individuals. It is possible, however, that although no gross changes in the species occur, the population may adapt to the presence of new substrates by a change in the biotypes present or in other ways.

Dietary fibre, which has a marked effect on the function of the large intestine, increasing faecal weight and reducing transit time (Eastwood \& Passmore, 1983), includes the plant gums widely used as thickening, gelling and emulsifying agents in the food industry. Previous work has shown that inclusion of guar gum in the diet of a human subject increased the total count of anaerobic bacteria in faeces (Bayliss \& Houston, 1985).

Gum arabic is the second most widely used gum in the food industry (Blenford, 1984) and as such forms part of the average Western diet, although at a low level. It is a highly water-soluble extract from Acacia senegal, and is a high-molecular-weight globular molecule with a backbone of D-galactopyranose units and complex side chains of D-galactose, $\mathrm{L}$-arabinose, L-rhamnose and D-glucuronic acid. The in vivo effects of gum arabic in the diet have been demonstrated by McLean Ross et al. (1983), who showed that breath hydrogen increased in subjects who had eaten a diet containing gum arabic for 3 weeks but not in control subjects whose diet had not contained gum arabic. It seems likely that this increase reflected an adaptation of the microbial population of the human colon to the presence of the substrate. Therefore, the present study was carried out to determine whether a specific change in the diet of a human volunteer could lead to a change in faecal flora or in that part of the flora associated with fermation of the new substrate. Enrichment techniques were used to isolate gum-arabic fermenters present in the faeces of two individuals.

* Present address; University of Strathclyde, Department of Bioscience and Biotechnology, Applied Microbiology Division, Royal College Building, 204 George Street, Glasgow G1 1XW. 


\section{MATERIALS AND METHODS}

Unless otherwise stated all media were prepared and manipulated under the rigorous anaerobic techniques of Hungate (1969).

\section{Gum arabic feeding trial}

A female volunteer ( $\mathrm{DH} 2$ ), age 32 years, who had not taken antibiotics during the previous 3 months, followed a $7 \mathrm{~d}$ rotating diet containing about $15 \mathrm{~g}$ dietary fibre/d, which had been assessed during a previous study (Bayliss \& Houston, 1985). During a treatment period, $10 \mathrm{~g}$ gum arabic (food grade; A. Branwell \& Co. Ltd, London) in $125 \mathrm{ml}$ dextrose solution $(70 \mathrm{~g} / 1)$ (Mclean Ross et al. 1983) was taken daily at 09.00 hours for $18 \mathrm{~d}$, in addition to the normal diet. The first dose of gum arabic was taken in conjunction with forty inert markers.

On the days indicated in Fig. 1 (p. 263), freshly-voided faecal samples were immediately placed in an anaerobic cabinet (atmosphere carbon dioxide-hydrogen - nitrogen (50:100:850 by vol.); Forma Scientific, Marietta, Ohio). Faecal slurries were prepared in anaerobic dilution solution (ADS; Bryant \& Burkey, 1953) as described previously (Bayliss \& Houston, 1985). Samples were taken for estimation of moisture content by drying to constant weight at $100^{\circ}$ and the remainder of the faecal sample was frozen at $-20^{\circ}$.

Viable anaerobic organisms were enumerated using a most-probable-number (MPN) method. The faecal slurries were further diluted in ADS and duplicate dilutions used to inoculate $(0.2 \mathrm{ml})$ five replicate tubes of SM10 medium, a complex medium which will recover a high proportion of faecal organisms (Barnes \& Impey, 1974). To estimate gum-arabic fermenters, similar inoculations were made into basal defined medium (no added carbohydrate; Bayliss \& Houston, 1984) with gum arabic (2 g/l) autoclaves in the medium (BDM + GA). Positive tubes were scored by visual inspection after incubation for 7 and $14 \mathrm{~d}$ at $37^{\circ}$, and counts were calculated by the method of Hurley \& Roscoe (1983). Growth in BDM + GA was visually sparse at the end-points of the MPN count and tubes which were doubtful positives were plated on VLhlf agar (Croucher et al. 1983) and incubated anaerobically for confirmation of growth.

\section{Analysis of carbohydrate in faeces}

Undegraded gum arabic in faeces gives a typical white precipitate when the supernatant fraction from a slurry is treated with acid alcohol (McLean Ross et al. 1983). Aqueous slurries of faeces $(3: 1, w / v)$ were centrifuged and the supernatant fractions treated $(1: 4$, $\mathrm{v} / \mathrm{v})$ with acid alcohol (50 ml $11.6 \mathrm{M}$-hydrochloric acid/l ethanol). A positive control was prepared by mixing a slurry with gum-arabic solution to give a final concentration of $50 \mathrm{~g}$ gum arabic/l. Additionally, an aqueous dilution series of gum arabic was prepared down to $1 \mathrm{~g} / 1$.

To estimate the total amount of residual carbohydrate in the faeces, portions of slurry were acid-hydrolysed and prepared for analysis by the method of Selvendran et al. (1979). Monosaccharides in the preparation were measured using a gas-liquid chromatograph (Perkin-Elmer; with a column packing of Supelco SP2330 3\% on 100/120 mesh Chromosorb) using inositol as an internal standard.

\section{Isolation and characterization of gum-arabic fermenters}

In a separate experiment before the feeding trial, freshly-voided faeces from $\mathrm{DH} 2$, and also from a male volunteer (DH1), were collected and sampled in the anaerobic cabinet. The initial slurry was diluted to $10^{-13}$, in triplicate, and incubated at $37^{\circ}$ in $10 \mathrm{ml}$ tubes of $\mathrm{BDM}+\mathrm{GA}$. When no further tubes showed visible growth, one tube of BDM + GA from 


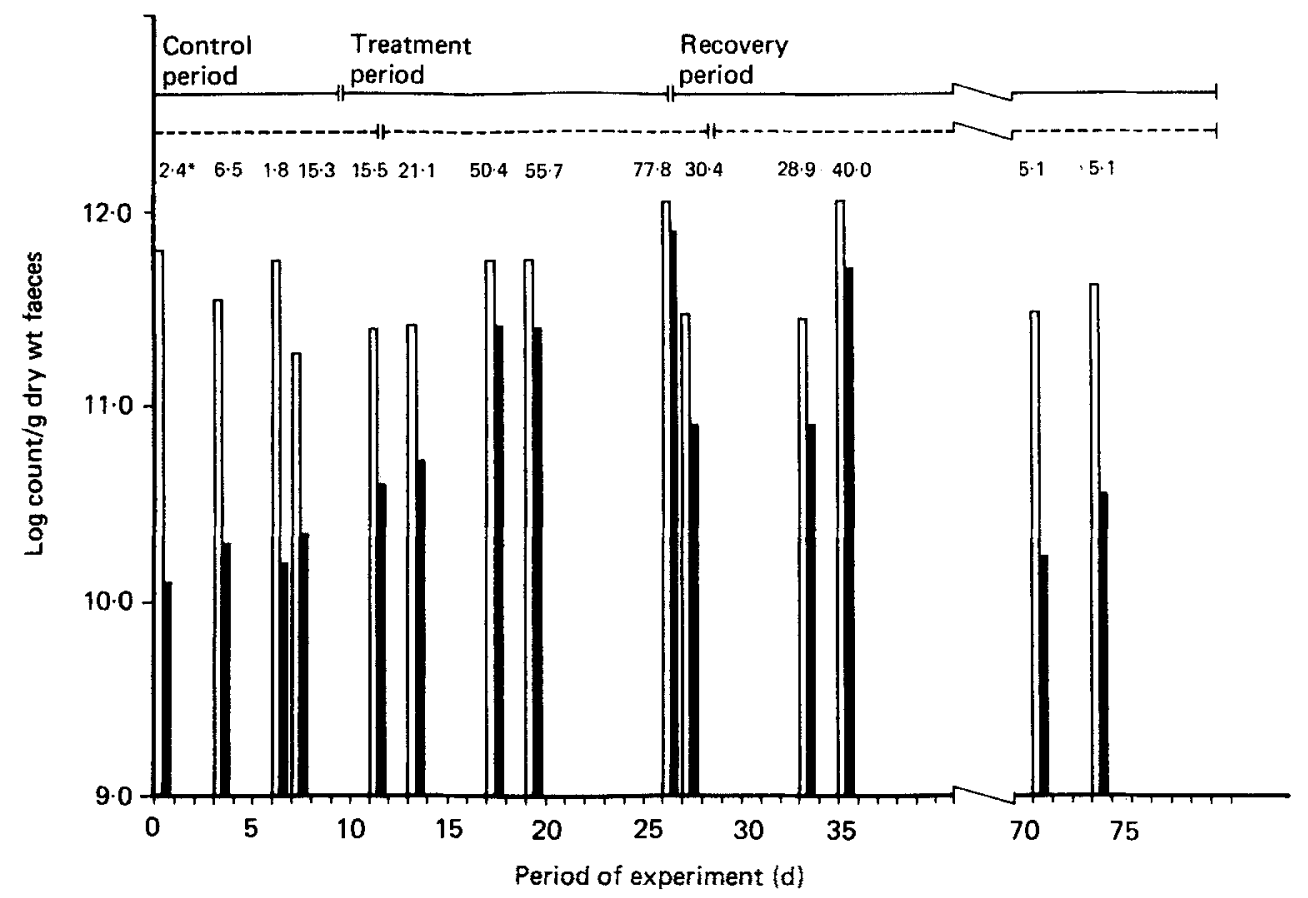

Fig. 1. Anaerobic viable counts of human faecal flora during a feeding experiment when gum arabic (GA) was included in the diet. ( - ), Actual feeding period; (---), adjusted for transit time; ( $\square$ ), SM10 medium; $(\square)$, basal defined medium (BDM) + GA. Values are means of duplicate counts on each sample. *Percentage fermenting GA (count in BDM + GA as percentage of count in SM10).

the highest dilution showing growth was diluted in ADS, plated onto VLhlf agar and incubated anaerobically at $37^{\circ}$. All the colonies from the top dilutions showing growth were subcultured and isolates were then tested for their ability to ferment gum arabic by subculturing into BDM +GA. Identification tests were carried out on representative gum-arabic-fermenting isolates as described by Bayliss \& Houston (1984).

\section{Ethical considerations}

The protocol for this work was approved by the Food Research Institute Ethical Committee.

\section{RESULTS}

\section{Effect of ingestion of gum arabic on bacterial counts}

Unlike guar gum (Bayliss \& Houston, 1985) where flatus production increased, no subjective effects of the gum-arabic diet were noted by the volunteer. The first markers from the initial dose of gum arabic appeared in a faecal sample approximately $47 \mathrm{~h}$ after ingestion. Total viable anaerobic counts (Fig. 1) showed no significant trend during the control (four counts), treatment (six counts) and recovery (four counts) periods, with the lowest and highest counts being $1.9 \times 10^{11}$ and $1.1 \times 10^{12} / \mathrm{g}$ dry weight of faeces respectively.

The proportion of bacteria able to ferment gum arabic (BDM + GA, Table 1) rose during the gum-arabic diet from about $6 \%$ to greater than $50 \%$ in the late treatment period, indicating adaptation of the flora. During the early part of the recovery diet, the proportion had decreased slightly and, $45 \mathrm{~d}$ after ingestion of the last gum arabic, it was the same as that before ingestion. 


\section{Effect of gum arabic ingestion on faeces}

The mean moisture content of the faeces was slightly higher on the gum-arabic diet (Table 1) but the difference was not statistically significant. Gum arabic was precipitable by acid alcohol from aqueous solution as a white aggregate at levels down to $1 \mathrm{~g} / 1$ and also from the faecal slurry to which gum arabic had been added. All faecal samples were tested but, apart from the gelatinous brown precipitate noted by McLean Ross et al. (1983), present in each sample, no other effect was seen.

Both total sugar content and the total of arabinose + rhamnose + galactose (which if present might indicate residual gum arabic) in hydrolyzed faecal samples were higher during the early treatment period when compared with the other periods (Table 1), but this difference was not statistically significant because of the large standard deviations involved.

\section{Characterization of gum-arabic fermenters}

Twenty-two colonies were originally picked from the VLhlf plates (eighteen from DH1 and four from $\mathrm{DH} 2$ ) and two isolates from each subject, representing the major colony morphologies, were characterized further. Isolates $\mathrm{DH} 1 / 8$ and $\mathrm{DH} 2 / 1$ were Gram-positive branching rods showing most of the reactions of Bifidobacterium longum; $\mathrm{DH} 1 / 9$ and DH2/2 were Gram-negative rods identified as Bacteroides ovatus and a member of the Bacteroides oris/Bacteroides buccae group respectively (Holdeman et al. 1984).

\section{DISCUSSION}

The work reported here shows that a specific change in the diet of a human volunteer, i.e. the addition of gum arabic to a normal Western diet, was accompanied by a rapid adaptation of the faecal flora, such that the proportion of bacteria able to ferment gum arabic increased markedly. After gum arabic was withdrawn from the diet the proportion of gum-arabic fermenters returned to the level present before ingestion (Fig. 1), confirming that the response was due to dietary change. Such effects have not previously been recognized, other studies having found that human faecal flora does not change in response to diet. In view of the importance of the colonic flora in health, for example in supplying volatile fatty acids which act as an energy supply for the colonic epithelium and in producing mutagens, the ability of the flora to alter rapidly in response to dietary change is of obvious significance.

The total viable count of anaerobes per $g$ dry weight of faeces present during the treatment period in the present study was unchanged, in contrast to the effect seen previously with guar gum (Bayliss \& Houston, 1985), where the total count per g dry weight of faeces increased seventeen-fold but the proportion of guar-gum fermenters stayed the same. Additionally, with guar gum total bacterial output was further raised by an increase in the daily faecal output. In the present study, total faecal collections were not made so it is not possible to determine what changes, if any, occurred in total bacterial excretion. Guar gum is much more viscous than gum arabic and, on addition to the diet of rats, may allow monosaccharides to pass to the large intestine (J. M. Gee and I. T. Johnson, personal communication) and might thus allow greater growth of organisms other than guar-gum fermenters.

The precipitation test for residual gum arabic was applied to the first faecal sample to contain markers but this, as well as the other samples tested, was negative. Clearly, the residence time of gum arabic in the large intestine, even with the lowest level of gumarabic fermenters found, was sufficient for the polymer to be degraded. Fragments of the polymer, if present, would contribute to the level of rhamnose, arabinose and galactose 
in acid-hydrolyzed faeces. These sugars were higher during the early treatment period, suggesting that gum arabic was not completely utilized at this time; however, this increase was not statistically significant due to the large day-to-day variation in faecal sugar levels, probably resulting from an incompletely-standardized diet.

This work has shown that a shift in the metabolic activity of the faecal flora can occur in response to a dietary change; further work is required to identify the organisms involved in this adaptive shift, measure changes in levels of relevant degrading enzymes in the faeces and to ascertain whether or not a similar effect occurs with other dietary fibres or in other subjects.

It seems clear that when examining the ability of gut organisms to degrade fibre, enrichment techniques can be advantageous because, although Salyers et al. (1977 $a, b)$ found little fermentation of gum arabic using faecal isolates, we readily obtained Bacteroides and Bifidobacterium spp. from faeces by our methods which were able to ferment gum arabic in pure culture. The plea by Tannock (1982) to monitor the whole collection of microbes in the intestinal tract in relation to changes in the host diet should be heeded.

The authors would like to thank Katy Hamand for skilled technical work, A. Branwell \& Co. for the gift of gum arabic and W. M. Waites for much helpful discussion and criticism. J.D.H. was a third year undergraduate student at the University of East Anglia during this work.

\section{REFERENCES}

Barnes, E. M. \& Impey, C. S. (1974). Journal of Applied Bacteriology 37, 393-409.

Bayliss, C. E. \& Houston, A. P. (1984). Applied and Environmental Microbiology 48, 626-632.

Bayliss, C. E. \& Houston, A. P. (1985). Food Microbiology 2, 53-62.

Blenford, D. E. (1984). Food 6 (7), 9.

Bryant, M. P. \& Burkey, L. A. (1953). Journal of Dairy Science 36, 205-217.

Croucher, S. C., Houston, A. P., Bayliss, C. E. \& Turner, R. J. (1983). Applied and Environmental Microbiology 45, 1025-1033.

Eastwood, M. A. \& Passmore, R. (1983). Lancet ii, 202-206.

Holdeman, L. V., Kelley, R. W. \& Moore, W. C. (1984). In Bergey's Manual of Systematic Bacteriology, vol. 1. pp. 602-631 [N. R. Krieg and J. G. Holt, editors]. Baltimore: Williams and Wilkins.

Hungate, R. E. (1969). In Methods in Microbiology, vol. 3B, pp. 117-132 [J. R. Norris and D. W. Ribbons, editors]. London: Academic Press.

Hurley, M. A. \& Roscoe, M. E. (1983). Journal of Applied Bacteriology 55, 159-164.

McLean Ross, A. H., Eastwood, M. A., Anderson, J. R. \& Anderson, D. M. W. (1983). American Journal of Clinical Nutrition 37, 368-375.

Moore, W. E. C., Cato, E. P., Good, I. J. \& Holdeman, L. V. (1981). In Banbury Report no. 7, Gastrointestinal Cancer, pp. 11-19 [W. R. Bruce, P. Correa, M. Lipkin, S. R. Tannenbaum and W. D. Wilkins, editors]. Cold Spring Harbor, NY: Cold Spring Harbor Laboratory.

Salyers, A. A., Vercellotti, J. R., West, S. E. H. \& Wilkins, T. D. (1977 a). Applied and Environmental Microbiology 33, 319-322.

Salyers, A. A., West, S. E. H., Vercellotti, J. R. \& Wilkins, T. D. (1977b). Applied and Environmental Microbiology 34, 529-533.

Savage, D. C. (1982). In Fibre in Human and Animal Nutrition, pp. 125-129 [G. Wallace and L. Bell, editors]. Wellington: Royal Society of New Zealand.

Selvendran, R. R., March, J. F. \& Ring, S. G. (1979). Analytical Biochemistry 96, 282-292.

Tannock, G. W. (1982). In Fibre in Human and Animal Nutrition, pp. 131-134 [G. Wallace and L. Bell, editors]. Wellington: Royal Society of New Zealand. 